\title{
Mesoscopic Theory of Granular Fluids
}

\author{
T.P.C. van Noije and M.H. Ernst \\ Instituut voor Theoretische Fysica, Universiteit Utrecht, Postbus 80006, 3508 TA Utrecht, The Netherlands
}

\author{
R. Brito and J. A. G. Orza \\ Facultad de Ciencias Físicas, Universidad Complutense, 28040 Madrid, Spain
}

(Received 18 February 1997)

\begin{abstract}
Using fluctuating hydrodynamics we describe the slow buildup of long range spatial correlations in a freely evolving fluid of inelastic hard spheres. In the incompressible limit, the behavior of spatial velocity correlations (including $r^{-d}$ behavior) is governed by vorticity fluctuations only and agrees well with two-dimensional simulations up to 50 to 100 collisions per particle. The incompressibility assumption breaks down beyond a distance that diverges in the elastic limit. [S0031-9007(97)03645-4]

PACS numbers: 46.10.+z, 05.20.Dd, 05.40.+j, 81.05.Rm
\end{abstract}

In the characterization of granular matter as an unusual solid, fluid, or gas by Jaeger et al. [1], this Letter addresses the granular gas regime, controlled by inelasticity, clustering [2], and collapse [3]. Clustering is a long wavelength, low frequency (hydrodynamic) phenomenon and inelastic collapse a short wavelength, high frequency (kinetic) phenomenon. In the granular gas regime, also called rapid granular flows, the dynamics is dominated by inelastic collisions. Here the methods of nonequilibrium statistical mechanics, molecular dynamics, kinetic theory, and hydrodynamics are most suitable for describing the observed average macroscopic behavior [2-7] and the fluctuations around it.

The lack of energy conservation makes the granular gas, whether driven or freely evolving, behave very differently from molecular fluids. The essential physical processes and detailed dynamics are described in [2,3] and references therein: the similarities and differences with molecular fluids; lack of separation of microscales and macroscales, not only because the grains themselves are macroscopic, but also because of the existence of intermediate intrinsic scales which are controlled by the inelasticity and are only well separated when the system is nearly elastic. A simple model which incorporates the inelasticity of the granular collisions consists of inelastic hard spheres (IHS), taken here of unit mass and diameter, with momentum conserving dynamics. The energy loss in a collision is proportional to the inelasticity parameter $\epsilon=1-\alpha^{2}$ where $\alpha$ is the coefficient of normal restitution.

For an understanding of what follows, we recall two important properties of the undriven granular gas: (i) the existence of a homogeneous cooling state (HCS) and (ii) its instability against spatial fluctuations. The hydrodynamic equations for an IHS fluid, started in a uniform equilibrium state with temperature $T_{0}$, admit an HCS solution (see, e.g., $[2,3,7]$ ) with a homogeneous temperature $T(t)$, described by $\partial_{t} T=-2 \gamma_{0} \omega T$. Here the collision frequency is $\omega(T) \sim \sqrt{T} / l_{0}$ with a mean free path $l_{0}$, given by the Enskog theory [8] for a dense system of hard disks or spheres $(d=2,3)$ and $\gamma_{0}=\epsilon / 2 d$. Then $T(t)=$ $T_{0} /\left[1+\gamma_{0} \omega\left(T_{0}\right) t\right]^{2}=T_{0} \exp \left(-2 \gamma_{0} \tau\right)$, where $\tau$ is the average number of collisions suffered per particle within a time $t$. It is found by integrating $d \tau=\omega(T(t)) d t$. Moreover, this HCS solution is linearly unstable once the linear extent $L$ of the system exceeds some dynamic correlation length, which increases with decreasing $\epsilon$, and is proportional to $l_{0}[1-7]$.

The dynamics of fluctuations, say, in density, $\delta n(\mathbf{r}, t)$, and flow field, $\mathbf{u}(\mathbf{r}, t)$, have hardly been studied $[2,4,7]$, in sharp contrast to the large number of publications about the average behavior. We note that fluctuations are absent in hydrodynamic as well as in Boltzmann-Enskog-type kinetic equations which are based on molecular chaos (mean field assumption). The objects of interest in this Letter are the spatial velocity and density correlations

$$
\begin{gathered}
G_{\alpha \beta}(\mathbf{r}, t)=\frac{1}{V} \int d \mathbf{r}^{\prime}\left\langle u_{\alpha}\left(\mathbf{r}+\mathbf{r}^{\prime}, t\right) u_{\beta}\left(\mathbf{r}^{\prime}, t\right)\right\rangle, \\
G_{n n}(\mathbf{r}, t)=\frac{1}{V} \int d \mathbf{r}^{\prime}\left\langle\delta n\left(\mathbf{r}+\mathbf{r}^{\prime}, t\right) \delta n\left(\mathbf{r}^{\prime}, t\right)\right\rangle,
\end{gathered}
$$

with $V=L^{d}$, and the structure factors $S_{\alpha \beta}(\mathbf{k}, t)$ and $S_{n n}(\mathbf{k}, t)$, which are the corresponding Fourier transforms. Goldhirsch et al. [2] initiated molecular dynamics studies of $S_{n n}(\mathbf{k}, t)$ and $S_{p p}(\mathbf{k}, t)=\sum_{\alpha} S_{\alpha \alpha}(\mathbf{k}, t)$, and related in a qualitative way the structure at small $k$ to the most unstable vorticity modes, and presented a nonlinear analysis to explain the enslaving of density fluctuations by the vorticity field [9]. A more quantitative description of the structure factors $S_{n n}(\mathbf{k}, t)$ [4] and $S_{p p}(\mathbf{k}, t)$ [7] has been recently proposed, based on the dynamics of macroscopic unstable modes (Cahn-Hilliard theory of spinodal decomposition [10]). However, numerical evidence from molecular dynamics for the quantitative validity of this theory is still lacking.

The main goal of the present Letter is to calculate the velocity correlation function $G_{\alpha \beta}(\mathbf{r}, t)$ in unforced granular flows and to show that fluctuating hydrodynamics [11] 
gives a quantitative description of the spatial correlations over a large intermediate time interval, controlled by linearized hydrodynamics. Because the flows in freely evolving IHS systems are approximately incompressible $(\nabla \cdot \mathbf{u}=0)$, the vorticity field, $\nabla \times \mathbf{u}$, in the nonlinear Navier-Stokes equations is practically decoupled from the other hydrodynamic fields in the system. This implies that the density $n$ and the temperature $T(t)=T_{0} \exp \left(-2 \gamma_{0} \tau\right)$ can be considered homogeneous, and an approximate theory based on vorticity fluctuations alone is justified.

Thus, we describe the Fourier modes of the vorticity field or transverse flow field $\mathbf{u}_{\perp}(t)$ by the mesoscopic Langevin equation [11] (valid for $k l_{0} \lesssim 1$ )

$$
\partial_{t} \mathbf{u}_{\perp}(\mathbf{k}, t)+\nu(T(t)) k^{2} \mathbf{u}_{\perp}(\mathbf{k}, t)=\hat{\mathbf{F}}(\mathbf{k}, t),
$$

where $\mathbf{u}_{\perp}$ and $\hat{\mathbf{F}}$ are orthogonal to $\mathbf{k}$, and where $\nu(T) \sim$ $l_{0} \sqrt{T}$ is the kinematic viscosity of the IHS fluid in the HCS. The random noise $\hat{\mathbf{F}}$ is assumed to be white and Gaussian with a correlation

$$
\left\langle\hat{F}_{\alpha}(\mathbf{k}, t) \hat{F}_{\beta}\left(-\mathbf{k}, t^{\prime}\right)\right\rangle / V=B_{\alpha \beta}(T(t)) k^{2} \delta\left(t-t^{\prime}\right),
$$

and a noise strength $B_{\alpha \beta}(T)=2 \delta_{\alpha \beta} T \nu(T) / n$ [11]. With the additional assumption (see [2,3]) that the IHS viscosity has the same functional form as for elastic hard spheres or disks, the solution of the proposed Langevin theory provides a detailed prediction for $G_{\alpha \beta}(\mathbf{r}, t)$ on hydrodynamic space $\left(r \gtrsim l_{0}\right)$ and time scales $\left[\tau \gtrsim 1 / \omega\left(T_{0}\right)\right]$ without any adjustable parameters.

We briefly indicate how this is done by calculating the structure factor $S_{\perp}(\mathbf{k}, t)=\left\langle\left|u_{\perp \alpha}(\mathbf{k}, t)\right|^{2}\right\rangle / V$. Here the subscript $\alpha$ is one of the $(d-1)$ equivalent transverse components of $\mathbf{u}_{\perp}$. We transform Eq. (2) into the standard Langevin equation with time independent noise strength and coefficients. This is done by the change of variables $\omega(T(t)) d t=d \tau, \mathbf{u}_{\perp}(\mathbf{k}, t)=\sqrt{T(t)} \mathbf{w}(\mathbf{k}, \tau)$ and $\hat{\mathbf{F}}(\mathbf{k}, t)=\omega(T(t)) \sqrt{T(t)} \hat{\mathbf{f}}(\mathbf{k}, \tau)$ and yields $\partial_{\tau} \mathbf{w}(\mathbf{k}, \tau)-$ $z_{\perp}(k) \mathbf{w}(\mathbf{k}, \tau)=\hat{\mathbf{f}}(\mathbf{k}, \tau)$, with a growth rate $z_{\perp}(k)=$ $\gamma_{0}\left(1-k^{2} \xi^{2}\right)$ and a noise strength $b_{\alpha \beta}=2 \delta_{\alpha \beta} \gamma_{0} \xi^{2} / n$. The dynamic correlation length $\xi \equiv \sqrt{\nu / \omega \gamma_{0}}$ is time independent and of order $l_{0} / \sqrt{\gamma_{0}}$. With the help of the relation $\left\langle\left|w_{\alpha}(\mathbf{k}, 0)\right|^{2}\right\rangle=V / n$, the structure factor is then found as

$$
S_{\perp}(k, t)=\frac{T(t)}{n}\left\{1+\frac{\exp \left[2 \gamma_{0} \tau\left(1-k^{2} \xi^{2}\right)\right]-1}{1-k^{2} \xi^{2}}\right\},
$$

which is valid for $k l_{0} \lesssim 1$. In the elastic limit $\left(\gamma_{0} \rightarrow 0\right)$ the standard form of fluctuating hydrodynamics and the fluctuation dissipation theorem are recovered. For $k \xi \lesssim 1$ this equation describes the new structure of the velocity correlations $G_{\alpha \beta}(\mathbf{r}, t)$ on length scales of order $2 \pi \xi$. At the end of the Letter we return to the predicted structure on the largest scales.

On the shortest scales $(r \rightarrow 0), \quad G_{\alpha \beta}(\mathbf{r}, t) \rightarrow$ $[T(t) / n] \delta_{\alpha \beta} \delta(\mathbf{r})$, caused by self-correlations of particles. As our theory describes only structure on the scale $r \gtrsim l_{0}$, we consider the equivalent function $G_{\alpha \beta}^{+}(\mathbf{r}, t)$ with the self-correlations substracted, which is regular at the origin. For the same reason the structure factor $S_{\alpha \beta}(\mathbf{k}, t)$ has a plateau value $\delta_{\alpha \beta} T(t) / n$ for $k \rightarrow \infty$, whereas $S_{\alpha \beta}^{+}(\mathbf{k}, t) \rightarrow 0$ in the same limit. The function $S_{\alpha \beta}^{+}(\mathbf{k}, t)$ is an isotropic tensor field, which can be decomposed into two independent scalar functions of $k=|\mathbf{k}|$, i.e., $S_{\alpha \beta}^{+}(\mathbf{k}, t)=\hat{k}_{\alpha} \hat{k}_{\beta} S_{\|}^{+}(k, t)+\left(\delta_{\alpha \beta}-\hat{k}_{\alpha} \hat{k}_{\beta}\right) S_{\perp}^{+}(k, t)$, where a caret denotes a unit vector. The incompressibility assumption implies then $u_{\|}(\mathbf{k}, t)=0$, and consequently $S_{\|}^{+}(k, t)=0 . \quad$ Similarly, $G_{\alpha \beta}^{+}(\mathbf{r}, t)=\hat{r}_{\alpha} \hat{r}_{\beta} G_{\|}^{+}(r, t)+$ $\left(\delta_{\alpha \beta}-\hat{r}_{\alpha} \hat{r}_{\beta}\right) G_{\perp}^{+}(r, t)$. Consider first the longitudinal spatial correlation $G_{\|}^{+}(r, t) \equiv\left[T(t) / n \xi^{d}\right] g_{\|}\left(r / \xi ; 2 \gamma_{0} \tau\right)$, which is given by

$$
g_{\|}(x, s)=\int \frac{d \mathbf{q}}{(2 \pi)^{d}} e^{i \mathbf{q} \cdot \mathbf{x}} \sin ^{2} \theta \frac{\exp \left[s\left(1-q^{2}\right)\right]-1}{1-q^{2}},
$$

where $\cos \theta=\hat{\mathbf{q}} \cdot \hat{\mathbf{x}}$. In the incompressible limit the transverse correlation function is given by $g_{\perp}(x, s)=$ $g_{\|}(x, s)+[x /(d-1)] \partial g_{\|}(x, s) / \partial x$ (see [11], Chap. 3). These functions can be expressed as integrals over simple functions. As an example, we quote the result for $d=2$ :

$$
g_{\|}(x, s)=\frac{1}{2 \pi x^{2}} \int_{0}^{s} d s^{\prime} \exp \left(s^{\prime}\right)\left[1-\exp \left(-x^{2} / 4 s^{\prime}\right)\right] .
$$

The transverse function $g_{\perp}(x, s)$ has a negative minimum; moreover, $g_{\|}(x, s)$ is positive for all $x, s, d$; there are algebraic tails $g_{\|}(x, s) \sim-(d-1) g_{\perp}(x, s) \sim x^{-d}$ with a correction term of $\mathcal{O}\left(\exp \left(-x^{2} / 4 s\right)\right)$. Similar algebraic tails occur in nonequilibrium stationary states in driven diffusive systems [12]. These functions have structure on hydrodynamic space and time scales where both $x=r / \xi$ and $s=2 \gamma_{0} \tau$ can be either large or small with respect to unity. At small inelasticity $\left(\gamma_{0} \rightarrow 0\right)$ the dynamic correlation length and mean free path $l_{0}$ are well separated. Details will be published elsewhere [13].

To verify the theory quantitatively, we have performed event-driven molecular dynamics simulations of smooth inelastic hard disks, using square periodic boundary conditions and $N=5 \times 10^{3}, 2 \times 10^{4}$, and $5 \times 10^{4}$ particles. The inelasticity parameter $\epsilon=1-\alpha^{2}$ was varied between 0.02 and 0.8 , and the area fraction from $\phi=0.02$ to 0.4 , far below the solid transition $\left(\phi_{\text {solid }}=\right.$ 0.665 ). Before considering the range of validity of our results, we show in Fig. 1(a) how the relative vorticity fluctuations $\mathbf{w}(\mathbf{k}, \tau)$ have grown [2,3,7]. The HCS and linear hydrodynamics start to break down. The vorticity field becomes large and evolves into a "dense fluid of closely packed vortex structures," which is still homogeneous on scales large compared to $L_{v}$. The larger the inelasticity parameter $\epsilon$, the more rapidly this happens.

Apart from the restrictions to hydrodynamic space and time scales, there are two essential criteria limiting the validity of our theory: (i) System sizes $L$ must be thermodynamically large $(L \gg 2 \pi \xi)$, so that Fourier sums over 

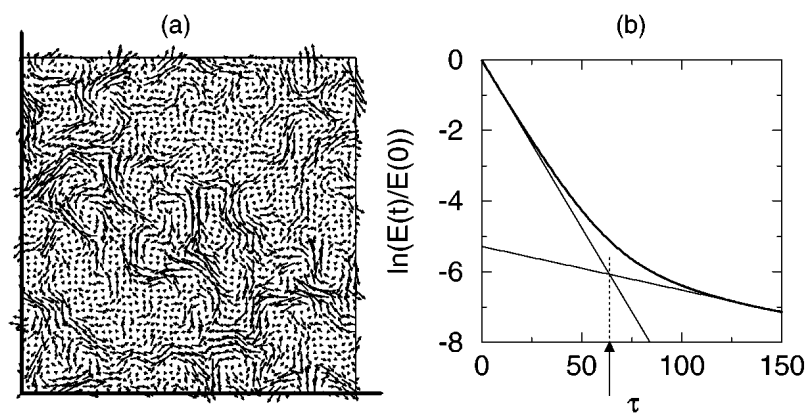

FIG. 1. (a) Snapshot of the momentum density at $\tau=80$ in a system with $N=5 \times 10^{4}, L=313(\phi=0.4), \alpha=0.9$, coarse grained over cells of $6.3 \times 6.3$, representing a rather stable (still very similar at $\tau=160$ ) configuration of vortex structures of typical diameter $L_{v}(t) \simeq 25\left(l_{0} \simeq 0.34\right)$ with nearest neighbors having opposite vorticity for reasons of stability. (b) $\ln [E(t) / E(0)]$ versus $\tau$ from the same simulation showing the linear (HCS) regime, where $E(t)=T(t)$, with slope $-2 \gamma_{0}$, up to a crossover time $\tau_{\mathrm{cr}} \simeq 65$, and the nonlinear cooling regime with a smaller slope, where the cooling through inelastic collisions is partially compensated by viscous heating.

$\mathbf{k}$ space can be replaced by $\mathbf{k}$ integrals. (ii) Times must be restricted to the linear hydrodynamic regime $\left(\tau \lesssim \tau_{\mathrm{cr}}\right)$, so that the system remains close to the HCS. Monitoring the energy per particle $E(t)$ provides a sensitive criterion to distinguish the linear from the nonlinear cooling regime, where the appearance of gradients causes viscous heating and slows down the cooling [2] [see Fig. 1(b)]. The crossover time $\tau_{\text {cr }}$ in Fig. 1(b), decreasing with increasing $\epsilon$ and $\phi$, is an intrinsic time scale that only depends on the existing gradients. At large $(\epsilon, \phi)$ values criterion (ii) reduces $\tau_{\mathrm{cr}}(\epsilon ; \phi)$ to subhydrodynamic time scales, with $\tau_{\mathrm{cr}}(0.5 ; 0.25) \simeq 15$ and $\tau_{\mathrm{cr}}(0.3 ; 0.4) \simeq 25$ as borderline cases. On the other hand, small $(\epsilon, \phi)$ values combined with small $L$ tend to violate criterion (i). The small systems with $N=5 \times 10^{3}$, and to some extent even those with $N=2 \times 10^{4}$, only satisfy the criteria (i) and (ii) in very narrow parameter ranges. The systems studied in Ref. [3] $(N=1024)$, [4] $(N=1600)$, and [7] $\left(N=5 \times 10^{3}\right)$ are, in large regions of parameter space, so small that $L$ is comparable to $2 \pi \xi$, and the periodic boundaries induce spurious transitions in the granular flows.

We have measured in simulations the equal time spatial correlation functions $G_{\mu}(\mathbf{r}, t)$ with $\mu=\{n n, \|, \perp\}$ by two methods, first by summing $a_{\mu}\left(\mathbf{v}_{i}\right) a_{\mu}\left(\mathbf{v}_{j}\right)$ over pairs of particles, where $a_{\mu}\left(\mathbf{v}_{i}\right)=\left\{1,\left(\mathbf{v}_{i} \cdot \mathbf{r}\right),\left(\mathbf{v}_{i} \cdot \mathbf{r}_{\perp}\right)\right\}$, and binning their relative position vectors into circular shells, and secondly by squaring the Fourier transform of the coarse-grained fields, followed by an inverse Fourier transformation. The second approach also provides the correlations in $\mathbf{k}$ space, which allows us to separate the contributions of $S_{\|}(k, t)$ and $S_{\perp}(k, t)$ to $G_{\|}(r, t)$ and to $G_{\perp}(r, t)$ and test the validity of the incompressibility assumption.

Figures 2(a)-2(c) show the longitudinal and transverse correlation functions of the flow field of a single simula- (a)
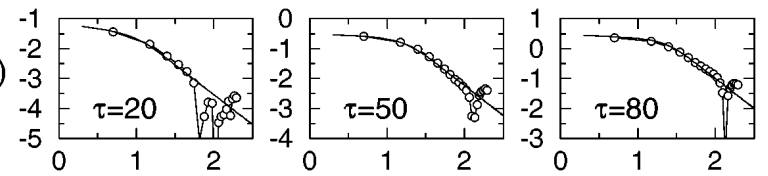

(b)
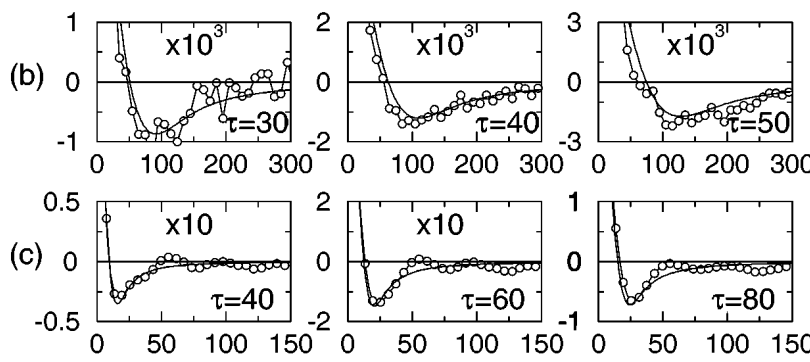

FIG. 2. Correlation functions $G_{\mu}(\mu=\{\|, \perp\})$ versus $r$ at various times, inelasticities and densities for $N=5 \times 10^{4}$ particles, where simulation results of a single run are compared with theoretical predictions (solid line). Top row (a) shows $\log _{10}\left[G_{\|} / T\right]$ versus $\log _{10} r$ at $\alpha=0.9$ and $\phi=0.245\left(l_{0} \simeq\right.$ 0.8 ), where $\tau_{\text {cr }} \simeq 70$. The algebraic $1 / r^{2}$-tail is clearly visible. Middle row (b) shows $G_{\perp} / T$ versus $r$ at $\alpha=0.94$ and $\phi=$ $0.05\left(l_{0} \simeq 5.8\right)$ where $\tau_{\mathrm{cr}} \simeq 100$. Bottom row (c) shows $G_{\perp} / T$ versus $r$ at $\alpha=0.9$ and $\phi=0.4\left(l_{0} \simeq 0.34\right)$ where $\tau_{\mathrm{cr}} \simeq 65$. Note regular oscillations with period $R_{0} \simeq 50$, which is fixed in time.

tion run at $N=5 \times 10^{4}$ at several $(\epsilon, \phi)$ values. The low noise levels, observed in these data and in Fig. 1(a), are a consequence of the IHS collisions which have the tendency to make particles move parallel. There is reasonable agreement with the theoretical predictions from our Langevin theory, in which the viscosity is taken from Enskog's theory. No fitting parameters are involved. The longitudinal $G_{\|}(r, t)$ in Fig. 2(a) shows good agreement for a large range of $(\epsilon, \phi)$ values, well beyond the linear time regime $\tau_{\mathrm{cr}}$. It exhibits the $1 / r^{2}$ tail. The minimum in $G_{\perp}(r, t)$ at $L_{v}(t)$ can be identified with the mean vortex diameter, and the low noise data in Fig. 2(c) at different $\tau$ show that $L_{v}(t) \sim \sqrt{\tau}$ is growing through vorticity diffusion. At small $(\epsilon, \phi)$ values $G_{\perp}(r, t)$ agrees well with our theory, as illustrated in Fig. 2(b). The $1 / r^{2}$ tail in $G_{\perp}$ cannot be observed in a single run because of statistical fluctuations. At larger densities [see Fig. 2(c)] one observes small oscillations around the predicted curve with a characteristic length $R_{0} \simeq 50$. The oscillations become more pronounced at later times, where $R_{0}$ stays fixed in time, but varies over different runs. Comparison of $G_{\perp}$ at $\tau=80$ with the snapshot in Fig. 1(a) at the same parameters suggests that $G_{\perp}$ may be viewed as the pair correlation function of a densely packed fluid of "hard objects" (vortices) of typical diameter $L_{v}$, the oscillation length $R_{0} \simeq 2 L_{v}$ being approximately equal to the size of a nearest neighbor $(+-)$ vortex pair. Similar complex structures, persisting in the nonlinear regime, are typically observed at larger $(\epsilon, \phi)$ values $\left(N=4 \times 10^{4}, \epsilon=0.64, \phi=0.05\right.$ [2]; $\quad N=5 \times 10^{4}, \epsilon \gtrsim 0.1, \phi \gtrsim 0.05 ; \quad N=2 \times 10^{4}$, $\epsilon>0.05, \phi>0.25$ ). At smaller $(\epsilon, \phi)$ values (linear 
(a)

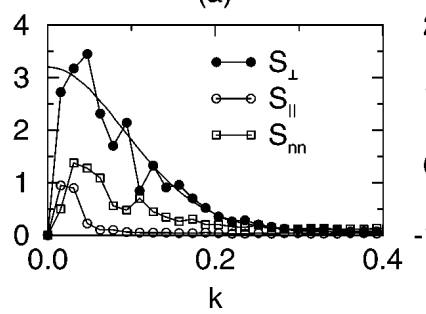

(b)

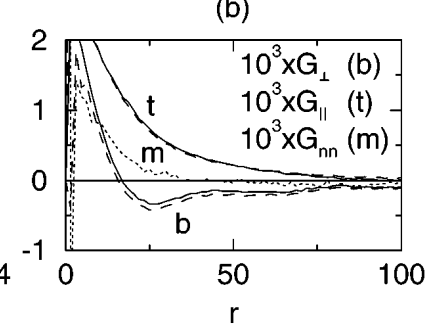

FIG. 3. (a) Structure factors $S_{\mu}(k, t)(\mu=\{\perp, \|, n n\})$ versus $k$ from a single simulation run at $N=5 \times 10^{4}, \alpha=0.9$, $\phi=0.245$ and $\tau=50$ where $\tau_{\text {cr }} \simeq 70$, compared with the theoretical prediction (solid line) for $S_{\perp}(k, t)$. All structure in $S_{\mu}(k, t)$ contained in the interval $k_{\min }=2 \pi / L \simeq 0.016<$ $k \lesssim 1 / \xi=0.25$ represents long range correlations of dynamic origin. (b) Correlation functions $G_{\mu}(r, t)(\mu=\{\|, \perp\})$ from $S_{\perp}$ and $S_{\|}$(solid line), and from $S_{\perp}$ only (dashed line). $G_{n n}(r, t)$ (dotted line) corresponds to $S_{n n}(k, t)$.

regime) the vortex diameter $L_{v}(t) \sim \sqrt{\tau}$, and a transition to a "sheared state" is induced by the periodic boundaries when $L_{v}(t) \simeq \frac{1}{2} L$ [7], for instance, at $\tau \simeq 600$ for $N=2 \times 10^{4}, \epsilon=0.05, \phi=0.245$.

The description of the velocity fluctuations $G_{\alpha \beta}(\mathbf{r}, t)$ in this Letter is based on fluctuating hydrodynamics for the vorticity fluctuations only, i.e., the absence of longitudinal fluctuations (incompressibility assumption). Figure 3(a) confirms that this assumption is very reasonable indeed, as $S_{\|}(k, t)$ is vanishingly small down to very small $k$ values $\left(k \geq 1 / \xi_{\|} \simeq 0.06\right)$. However, for the smallest wave numbers, the incompressibility assumption breaks down. In that range the longitudinal velocity fluctuations couple to the second unstable mode $[3,4,7]$ with a dispersion relation (to second order in $k$ ) $z_{\|}(k)=\gamma_{0}(1-$ $\left.k^{2} \xi_{\|}^{2}\right)$. The nonvanishing contributions of $S_{\|}(k, t)$ to $G_{\alpha \beta}(t)$ cause an exponential cutoff on length scales $r \geqslant$ $2 \pi \xi \| \gg 2 \pi \xi$, and the algebraic decay $\sim 1 / r^{d}$ from the vorticity mode represents intermediate behavior, which is well observable because the two length scales $\xi_{\|}$and $\xi$ are in general quite different, e.g., $\xi_{\|} \simeq 4.3 \xi$ in Figs. 2(a) and 2(c) and $\xi_{\|} \simeq 4.5 \xi$ in Fig. 2(b), and rapidly separate in the elastic limit.

The results of Fig. 3(a) were obtained by fast Fourier transformation of the density and momentum fields (coarse-grained into $256 \times 256$ cells), and performing an angular average in $\mathbf{k}$ space. In the same figure one observes that $S_{\|}(k, t)$ has the smallest width, while $S_{\perp}(k, t)$ and $S_{n n}(k, t)$ have a comparable width. Moreover, the growth rate of $S_{\perp}(k, t) / E(t)$ exceeds that of $S_{\|}(k, t) / E(t)$, which is in turn more unstable than $S_{n n}(k, t)$. Finally, if one performs an inverse Fourier transform on the measured $S_{\perp}(k, t)$ and $S_{\|}(k, t)$ separately to obtain the contributions to $G_{\|}(r, t)$ and $G_{\perp}(r, t)$ [see Fig. 3(b)], it appears that the contributions from $S_{\|}(k, t)$ are small, and our description of the fluctuations in terms of a Langevin equation based on incompressibility is confirmed by the simulations in the linear regime $\tau<\tau_{\mathrm{cr}}$.

The authors wish to thank H.J. Bussemaker, D. Frenkel, M. Hagen, and W.v.d. Water for helpful comments and discussions. T. v. N. acknowledges support of the foundation "Fundamenteel Onderzoek der Materie (FOM)," which is financially supported by the Dutch National Science Foundation (NWO). R. B. acknowledges support from DGICYT (Spain) No. PB94-0265.

[1] H. M. Jaeger, S. R. Nagel, and R. P. Behringer, Rev. Mod. Phys. 68, 1259 (1996).

[2] I. Goldhirsch and G. Zanetti, Phys. Rev. Lett. 70, 1619 (1993); I. Goldhirsch, M-L. Tan, and G. Zanetti, J. Scient. Comp. 8, 1 (1993); N. Sela, I. Goldhirsch, and S. H. Noskowicz, Phys. Fluids 8, 2337 (1996).

[3] S. McNamara, Phys. Fluids A 5, 3056 (1993); S. McNamara and W. R. Young, Phys. Rev. E 53, 5089 (1996).

[4] P. Deltour and J.-L. Barrat, J. Phys. I (France) 7, 137 (1997).

[5] S. E. Esipov and T. Pöschel, J. Stat. Phys. 86, 1385 (1997).

[6] J. J. Brey, F. Moreno, and J. W. Dufty, Phys. Rev. E 54, 445 (1996).

[7] J. A. G. Orza, R. Brito, T. P. C. van Noije, and M. H. Ernst, Report No. cond-mat/9702029.

[8] S. Chapman and T. G. Cowling,The Mathematical Theory of Non-uniform Gases (Cambridge University Press, Cambridge, 1970).

[9] The same nonlinear analysis of [2] reveals that the length scale $\xi \sim l_{0} / \sqrt{\epsilon}$, associated with clustering, is the same as the correlation length $\xi$ introduced above Eq. (4).

[10] J. S. Langer, in Solids Far from Equilibrium, edited by C. Godrèche (Cambridge University Press, Cambridge, 1992), p. 297.

[11] L. Landau and E. M. Lifshitz, Fluid Mechanics (Pergamon Press, New York, 1959), Chaps. 3 and 17.

[12] G. Grinstein, D.-H. Lee, and S. Sachdev, Phys. Rev. Lett. 64, 1927 (1990); B. Schmittmann and R. K.P. Zia, Statistical Mechanics of Driven Diffusive Systems (Academic Press, New York, 1995).

[13] R. Brito, J. A. G. Orza, T. P. C. van Noije, and M. H. Ernst (to be published). 